\title{
Spontaneous microencapsulation of geraniol by zein
}

\author{
I. V. W Ferreira, W. W. Focke*, E. L. du Toit \\ Institute of Applied Materials, Department of Chemical Engineering, University of Pretoria, Private Bag X20, \\ 0028 Hatfield, Pretoria, South Africa
}

Received 30 March 2018; accepted in revised form 5 June 2018

\begin{abstract}
Geraniol was successfully micro-encapsulated with zein in a one-step phase separation process in which the zein and geraniol were both initially dissolved in a water-ethanol mixture. Microcapsules were only formed at geraniol-to-zein mass ratios above unity. This is ascribed to the relative solubility of the oil and protein in the solvent at the point of incipient phase separation: The geraniol must phase separate first before zein precipitation commences. The resultant oil droplets subsequently provide the interfacial surfaces that act as nucleation sites for the precipitation of the amphoteric zein molecules on continued dilution with water. The near spherical microcapsules formed under these conditions, comprised a foamed closed-cell wall that enclose large internal cavities in which the geraniol was trapped. The highest particle yield, $85.3 \%$, and oil encapsulation efficiency, $83.5 \%$, were obtained at a geraniol to zein mass ratio of $3: 1$. The particle size distribution for this system was log-normal with $d_{10}, d_{50}$ and $d_{90}$ values of 10,32 and $88 \mu \mathrm{m}$ respectively. The temperature dependent geraniol release rate from these capsules followed power law kinetics with a release exponent of 0.80 .
\end{abstract}

Keywords: biodegradable polymers, microencapsulation, controlled release, repellent

\section{Introduction}

The overuse of pesticides on agricultural crops remains a matter of concern. Their high toxicity and non-biodegradable properties result in harmful residues not only in crops, but also in soil and water resources [1]. Alternative pest management strategies with a lower impact on human health and the environment are subsequently encouraged. A growing body of research has shown that essential oils can be used as an alternative to synthetic pest control products [2]. Active components (mainly lipophilic) in these oils can act as toxins [3], as well as feeding and oviposition deterrents to a wide variety of insect pests [4]. The major challenge, concerning the use of essential oils for pest management, is the sustained release of the active ingredients over an extended time period. Unfortunately many essential oil components also show a lack of stability of when exposed to environmental conditions [5]. Furthermore, their high volatility necessitates relatively high application rates and/or frequent re-application when applied in emulsion form [6]. This renders their use for large scale agricultural applications both unpractical and uneconomical. Encapsulation of volatiles in a polymer shell $[7,8]$ is a well-known technique to manage controlled release and protection of active ingredients. In addition, encapsulated oils may be easier to handle and, depending on the solubility of the encapsulating polymer, they can be dispersed as water-based formulations [9].

In this work, geraniol was chosen as a representative essential oil component for encapsulation in the biopolymer, zein. Geraniol is an acyclic monoterpene alcohol and a major component in various essential oils, e.g. rose-, palmarosa- and citronella oil. It is known to exhibit insecticidal and repellent properties and can be used as a natural pest control agent with low toxicity [10]. Zein is a prolamin-rich protein, 
found in the endosperm of the corn kernel (Zea Mays L.). Although it is approved for oral use by the Food and Drug Administration, it has poor nutritional value, since it is deficient in essential amino acids [11]. Furthermore, the high percentage of non-polar amino acids, results in an inherent hydrophobicity and is responsible for its unique solubility behaviour [12]. It is insoluble in water, but readily dissolves in aqueous mixtures of low aliphatic alcohols, e.g. ethanol and methanol. Subsequently, it can easily be converted into colloidal particles by simply changing the solubilizing capacity of the primary solvent through dilution with water [13] in a process referred to as anti-solvent precipitation or phase separation. Several investigators exploited this behaviour to encapsulate essential oil components using zein [1417]. However, all the capsules produced using this technique were in the nano-sized particle range. For industrial controlled release applications of the nature targeted in this work, larger particles, i.e. in the micrometre size range, would be more suitable [18]. In addition, relatively large amounts of oil can be contained in such structures [19]. The aim of this work was therefore to specifically produce micronsized zein-based core-shell particles that act both as reservoirs and as controlled release devises for the chosen essential oil component.

A one-step phase separation method with aqueous ethanol as solvent was used to produce the capsules. The geraniol to zein ratio was varied to show that micron-sized capsules can be produced if the relative solubility of the components in the solvent phase is considered. The morphology of successfully produced capsules was characterized and the temperature dependent release kinetics of geraniol was measured and modelled.

\section{Experimental}

\subsection{Materials}

Anhydrous ethanol [CAS No. 64-17-5] (99.9\%) were obtained from Merck (South Africa). Geraniol (trans-3,7-dimethyl-2,6-octadien-1-ol) [CAS No. 106-24-1] of purity 98\%, zein [CAS No. 9010-66-6] Product No. Z3625, and fluorescein isothiocyanate (FITC) [CAS No. 27072-45-3] were supplied by Sigma-Aldrich (South Africa).

\subsection{Solubility measurements}

In order to confirm phase separation boundaries reported in literature $[11,20]$, the solubility of both zein and geraniol in aqueous ethanol mixtures was measured in a temperature controlled laboratory $\left(22 \pm 2^{\circ} \mathrm{C}\right)$. Zein solubility was gravimetrically determined. Approximately $1.5 \mathrm{~g}$ of ethanol was used in all experiments and the amount of water was varied in order obtain the desired ethanol concentration. Zein (between 300 and $600 \mathrm{mg}$ ) was dissolved under continuous shaking at $200 \mathrm{rpm}$ on a laboratory scale orbital shaker for $3 \mathrm{~h}$. Undissolved zein was separated from the mixture under vacuum filtration using Whatman Grade 2 filter paper which was weighed after drying. Zein that settled at the bottom of the pre-weighed sample container was also measured after drying. Duplicate experiments were performed at each ethanol fraction investigated. Geraniol solubility was measured using a cloud point titration technique. Here geraniol was added in $\sim 10 \mathrm{mg}$ increments to $1 \mathrm{~g}$ of each of the aqueous ethanol concentrations. The samples were manually shaken after each addition. Development of cloudiness in the solution was used to mark oil phase separation.

\subsection{Preparation of microcapsules}

In all experiments, a $15 \%(\mathrm{w} / \mathrm{w})$ zein solution was prepared by dissolving $2 \mathrm{~g}$ of zein in an $80 \%(\mathrm{w} / \mathrm{w})$ aqueous ethanol solution. The geraniol, at geraniol to zein mass ratios $(\xi)$ between $4: 1$ and $1: 3$, was subsequently mixed into the zein solution. No phase separation of either the oil or the zein occurred and a homogenous liquid mixture was formed at all geraniol levels investigated. Phase separation was induced by the sudden dilution of the solvent with enough distilled water to ensure that the final solvent concentration is outside the zein solubility envelope ( $\approx 25 \%$ ethanol on a geraniol and zein free basis). The mixture was continuously agitated in a high speed commercial blender during water addition, after which agitation was continued for one more minute. Due to the low density of geraniol $\left(\rho_{\mathrm{g}}=\right.$ $0.879 \mathrm{~g} \cdot \mathrm{cm}^{-3}$ ) and the relatively large particle size, oil-containing microcapsules floated to the top of the liquid within minutes after agitation was stopped. Unutilised zein precipitated and collected at the bottom of the container $\left(\rho_{z}=1.25 \mathrm{~g} \cdot \mathrm{cm}^{-3}\right)$. Most of the oil containing particles were removed from the surface with a spatula, while the remainder were recovered from the solution through vacuum filtration on Whatman Grade 6 filter paper. The recovered microcapsules were stored at $-20^{\circ} \mathrm{C}$ for further analysis. Five samples of each geraniol-to-zein mass ratios 
were prepared. Two of these samples were lyophilised for $24 \mathrm{~h}$ at pressures between 100 and 400 mTorr.

\subsection{Particle yield}

The recovered microcapsules, from three different sample preparations, were placed in prior weighed Petri dishes and placed in an oven at $50^{\circ} \mathrm{C}$ for approximately one hour at which point they appeared tangibly dry. It was assumed that this procedure effectively removed all residual water, ethanol and non-encapsulated geraniol and that a negligible amount of encapsulated geraniol was lost. This was confirmed by kinetic studies. The subsequent particle mass was used to determine the particle yield according to Equation (1):

Particle yield $=$

$=\frac{\text { Mass of dried particles recovered }}{\text { Mass of geraniol }+ \text { mass of zein initially added }}$

Three samples of each geraniol-to-zein mass ratio were used for particle yield determination.

\subsection{Encapsulation efficiency}

Two samples of each geraniol-to-zein ratio where high particle yields were recorded were used for encapsulation efficiency measurements. Encapsulation efficiencies and zein participation efficiencies were determined through gravimetric analysis using a Perkin Elmer 4000 thermogravimetric analyser (TGA). TGA analysis was performed prior to and after lyophilization. Approximately $30 \mathrm{mg}$ samples of geraniol-containing microcapsules were placed in $50 \mu \mathrm{L}$ alumina pans. The samples were heated at a rate of $10^{\circ} \mathrm{C} \cdot \mathrm{min}^{-1}$ from 30 to $300^{\circ} \mathrm{C}$. The TGA chamber was continuously purged with nitrogen flowing at $50 \mathrm{~mL} \cdot \mathrm{min}^{-1}$. The inert atmosphere was chosen to prevent the oxidation of the zein and geraniol that might otherwise have affected the observed mass losses. TGA response curves of the pure components, i.e. water, ethanol, geraniol, and zein, were obtained using the same procedure. A comparison between response profiles of pure components and that of the microcapsules was used to determine the temperature range where mass loss of encapsulated geraniol occurred. This was subsequently used to estimate the oil encapsulation efficiency as the mass of encapsulated oil compared to the mass of geraniol initially added to the solution by using Equation (2):
Encapsation efficiency $=$

$=$ Oil fraction $\cdot \frac{\text { Particle yield }}{100} \cdot \frac{\xi+1}{\xi}$

Similarly, the zein participation efficiency was defined as the mass \% of zein utilized in microcapsule formation. Encapsulation efficiencies were determined both prior to and after lyophilization of the samples.

\subsection{Particle size analysis}

Particle size analysis was performed using a Malvern Mastersizer 3000 instrument. Non-lyophilised particles were dispersed in water and the concentration was adjusted to ensure an obscuration of $\sim 12 \%$. A particle refractive index of 1.52 was used. Three analyses were performed on each sample.

\subsection{Imaging by scanning electron microscopy and confocal microscopy}

A Zeiss Ultra 55 FESEM Field emission scanning electron microscope (FESEM) was used to study the morphology of lyophilized microcapsules at an acceleration voltage of $1 \mathrm{kV}$. The samples were coated with carbon prior to analysis.

Confocal microscopy was conducted on samples prior to and after lyophilization to confirm the particle structure that was observed for the freeze-dried samples. Fluorescein isothiocyanate (FITC) dissolved in water $\left(100 \mathrm{mg} \cdot \mathrm{L}^{-1}\right)$ was added to the microcapsules in order to induce fluorescence of the polymer in the structures. The mixture was placed on a microscopy slide and left for approximately three minutes to react. The confocal images were taken with a Zeiss LSM 510 Meta confocal microscope, equipped with a $100 \times$ oil immersion objective.

\subsection{Release kinetics}

The geraniol release kinetics for particles formed using $\xi=3$ was measured by isothermal thermogravimetric analysis (TGA) on a Perkin Elmer 4000 instrument according to the method adapted from [21]. Samples weighing $30 \mathrm{mg}$ were placed in $50 \mu \mathrm{L}$ alumina pans. Isothermal runs were conducted at 60,70 and $80^{\circ} \mathrm{C}$. The TGA chamber was continuously purged with nitrogen at $50 \mathrm{~mL} \cdot \mathrm{min}^{-1}$ to ensure that it approached sink conditions and the samples were kept in the oven until no further mass loss could be detected. An additional run was performed at $40^{\circ} \mathrm{C}$ in a convection oven. Silica gel was placed in the 
oven to ensure that low humidity conditions, similar to those in the TGA chamber, were maintained.

\section{Results and discussion}

\subsection{Solubility results}

The solubility data for zein and geraniol respectively are presented in Figure 1a. Also shown are phase separation data from Li et al. [20] and Shukla and Cheryan [11] for geraniol and zein respectively. Geraniol solubility measurements are in good agreement with previously reported values in terms of concentration as well as the shape of the phase separation boundary. The viscous nature of the solutions at higher zein concentrations reduced the reliability of the solids separation technique used in this work. Subsequently only the low concentration range of the zein phase envelope could be determined reliably. In this range, the solubility measurements agreed with solubility data reported by Shukla and Cheryan [11]. Therefore the literature values were used to indicate the zein phase boundary in further analyses.

\subsection{Particle yield}

Particle yield values at the different geraniol to zein ratios $(\xi)$ are reported in Figure 2. Also shown is the actual appearance of samples after phase separation. An abrupt increase in particle yield is observed at $\xi>1$. This coincides with the appearance of a distinct

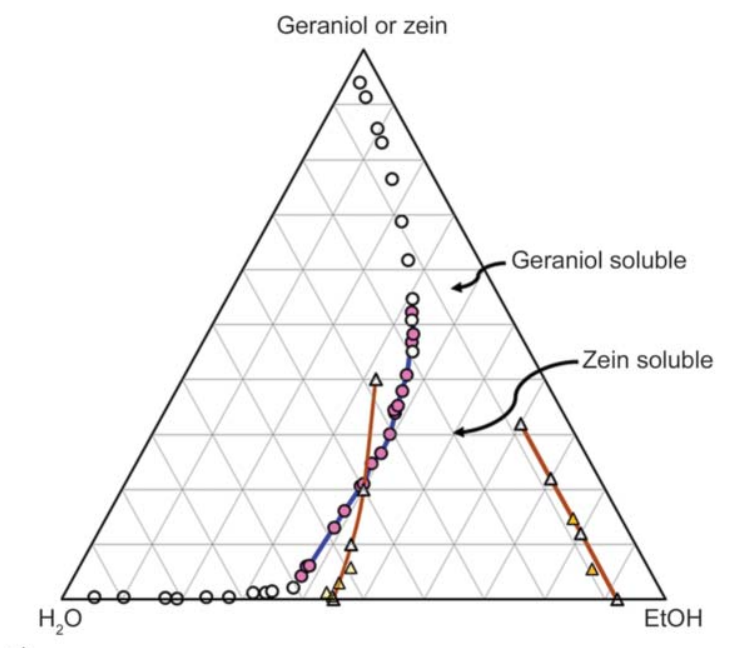

a)
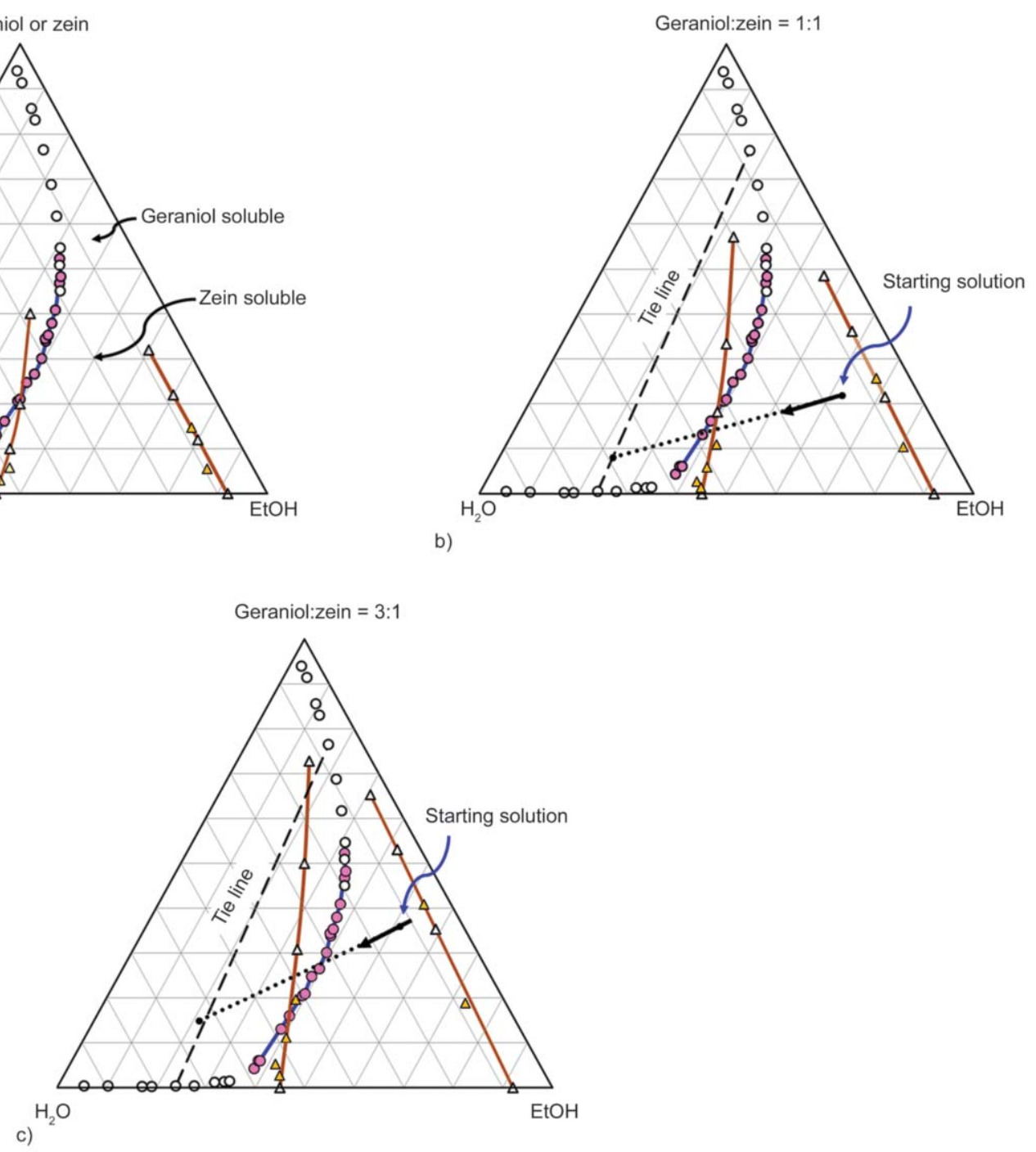

Figure 1. (a) Ternary diagram showing the solubility envelopes in aqueous ethanol solutions. Open circles represent data from Li et al. [20] while open triangles represent the zein phase envelope given by Shukla and Cheryan [11]. Filled circles and triangles show the data from this work. (b) Ternary diagram with zein phase envelope normalized to account for geraniol in the mixture at $\xi=1$. (c) Ternary diagram with zein phase envelope normalized to account for geraniol in the mixture at $\xi=3$. The normalized starting solution as well as the water dilution locus lines are also indicated on (b) and (c). Along the arrows, the solutions remain homogeneous but they point to the onset of phase separation. Tie lines in the geraniol phase envelopes are from Li et al. [20]. 
creamed particle layer. No oil layer was visible in these experiments and it was easy to disperse the particles by gently agitation. Precipitated zein at the bottom of the containers where low particle yields were measured, can be distinguished by the darker yellow colour of this layer. The highest particle yield $(85.3 \%)$ was obtained with $\xi=3$.

The kinetics of the precipitation process may explain the particle yield results. According to Filippidi et al. [19] the mechanism of polymer shell formation involves nucleation of zein at an oil interface followed by shell growth by zein precipitation from the bulk ethanol water phase. This implies that the particle yield results can be understood in terms of the relative positions of the phase envelopes and the composition locus that is followed when the mixture is diluted with water. To visualize the relative positions where phase separation would occur, the zein phase boundary was normalized to account for the geraniol present in the different formulations. Figure $1 b$ and $1 c$ show the adapted ternary diagrams for $\xi=1$ and $\xi=3$ respectively. The dilution locus follows a straight line connecting the starting composition with the final solution composition after phase separation. Note that the relative position of the phase separation boundaries is different between these two cases. For $\xi=1$, the saturation line for zein is reached first and it will precipitate out of the solution as zein particles and
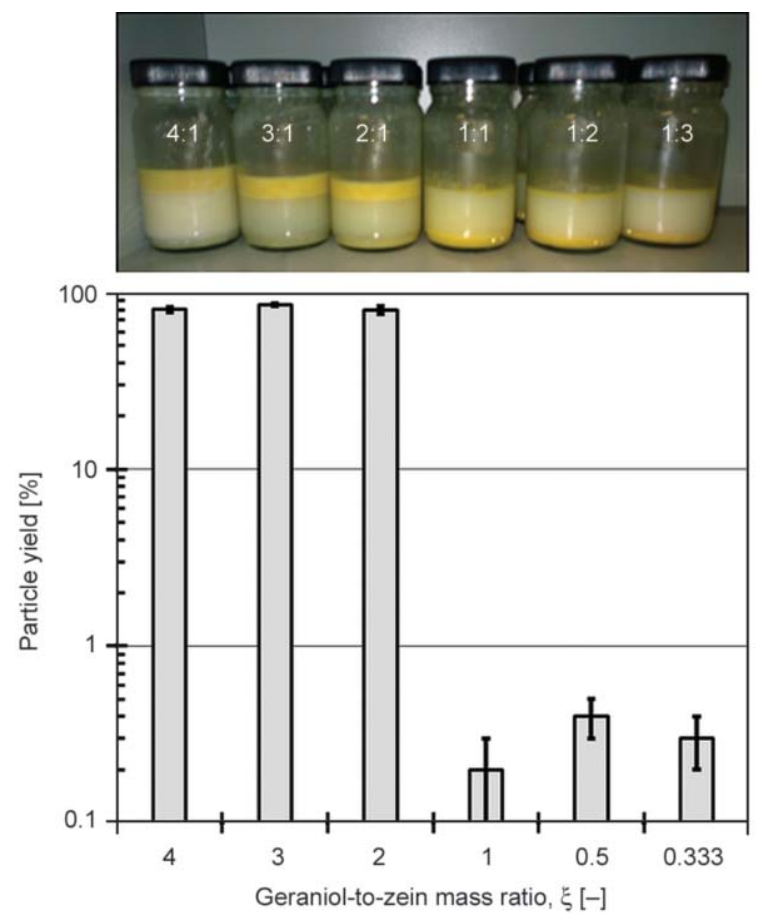

Figure 2. The effect of the geraniol-to-zein mass ratio $(\xi)$ on particle yield. consequently only very limited microencapsulation is achieved. However, for $\xi=3$ the phase separation of geraniol precedes that of the zein. The surfaces of the small geraniol oil droplets provide the necessary nucleating cites for zein precipitation. This happens once its solubility limit is exceeded by the continued dilution. Subsequently, the zein assembles as a layer that covers and coats the oil droplets to form the walls of the microcapsules that contain the geraniol. In other studies, essential oil components were encapsulated by zein with assistance of surfactants but relatively low oil to zein ratios as well as lower initial zein concentrations were used [14-17]. The oil to zein ratio ranged from $1: 4$ and $1: 2$ in solutions containing from 0.02 to $6.25 \%(\mathrm{w} / \mathrm{w})$ zein. The formed capsules were all in the nano-particle range with $d_{50} \leq 250 \mathrm{~nm}$. In those studies a different particle formation process, where nano-sized precipitated zein particles agglomerate at the surface of very small oil droplets, may have occurred. This can explain the particle morphology described by Parris et al. [14], where agglomerates of smaller nano-sized particles were observed.

\subsection{Particle size analysis}

Particle size distributions are shown in Figure 3 and summarized in Table 1. They feature strong peaks consistent with a log-normal distribution, i.e. they are slightly skewed towards smaller particle sizes. Noteworthy is the smaller size of the microcapsules obtained for the geraniol-to-zein mass ratio of $\xi=3$, which also had the highest particle yield. In the thin-wall limit, and assuming full utilization of both the zein and the geraniol in the formation of

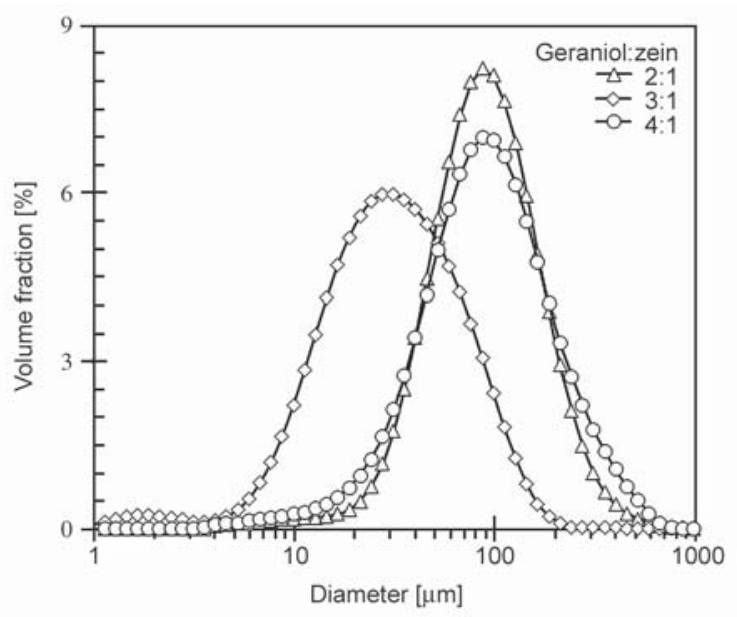

Figure 3. The effect of geraniol-to-zein mass ratio on particle size distribution. 
Table 1. Particle sizes and theoretical wall thickness estimates.

\begin{tabular}{|c|c|c|c|c|}
\hline $\begin{array}{c}\text { Geraniol-to-zein } \\
\text { mass ratio, } \boldsymbol{\xi}_{\mathbf{0}} \\
{[-]}\end{array}$ & \multicolumn{3}{|c|}{$\begin{array}{c}\text { Particle size } \\
{[\boldsymbol{\mu m}]}\end{array}$} & $\begin{array}{c}\text { Wall } \\
\text { thickness, } \boldsymbol{t}^{*} \\
{[\boldsymbol{\mu} \mathbf{m}]}\end{array}$ \\
\cline { 2 - 5 } & $\boldsymbol{d}_{\mathbf{1 0}}$ & $\boldsymbol{d}_{\mathbf{5 0}}$ & $\boldsymbol{d}_{\mathbf{9 0}}$ & 2.8 \\
\hline $2: 1$ & $40.7 \pm 0.0$ & $93.1 \pm 0.3$ & $206 \pm 1$ & 1.2 \\
\hline $3: 1$ & $10.4 \pm 0.2$ & $31.9 \pm 0.1$ & $88 \pm 1$ & 5.5 \\
\hline $4: 1$ & $34.3 \pm 0.4$ & $95.2 \pm 0.4$ & $251 \pm 12$ & \\
\hline
\end{tabular}

*Values estimated from Equation (3) and $d_{50}$ values.

the microcapsules, the thickness of the membrane wall is given by Equation (3):

$t=\frac{\rho_{\mathrm{g}} d}{6 \rho_{\mathrm{z}} \xi}$

Where $t$ is the capsule wall thickness, $d$ is the diameter of the capsule, $\rho_{\mathrm{g}}$ and $\rho_{\mathrm{z}}$ are the effective densities of the geraniol and zein respectively, and $\xi$ is the geraniol-to-zein mass ratio. Equation (3) indicates that the membrane wall thickness should scale linearly with the capsule diameter and inversely with the geraniol-to-zein ratio. Table 1 lists predicted values based on $d_{50}$. It shows that the predicted wall thickness for $\xi=3$ is about $1.2 \mu \mathrm{m}$.

\subsection{Encapsulation efficiency}

The TGA response curves of capsules formed at geraniol-to-zein mass ratios of $\xi=1,2$ and 3 are given in Figure 4. In Figure 4a the mass loss curves of the particles before and after lyophilization are shown together with the mass loss curves of the individual components. By considering the profiles of the pure components, the mass loss between 135 and $270{ }^{\circ} \mathrm{C}$ - marked by the vertical serrated lines - was associated with the geraniol content of the sample.

The derivative weight curves of the TGA response of particles prior to lyophilization - solid lines on Figure 4b, show three main peaks. They correspond to the volatilization of the ethanol (endotherm peak at the lowest temperature), water (middle peak), and geraniol (peak at the highest temperature). These temperature ranges can also be identified by considering the inflection points on the mass loss response curves in Figure 4a. The sharp peaks at temperatures between 130 and $140^{\circ} \mathrm{C}$ can be explained by considering the liquid-liquid-equilibrium (LLE) of the geraniol system. According to the tie line [20] in the geraniol phase envelope, marked in Figure 1b and 1c, the encapsulated oil rich phase will also contain a small
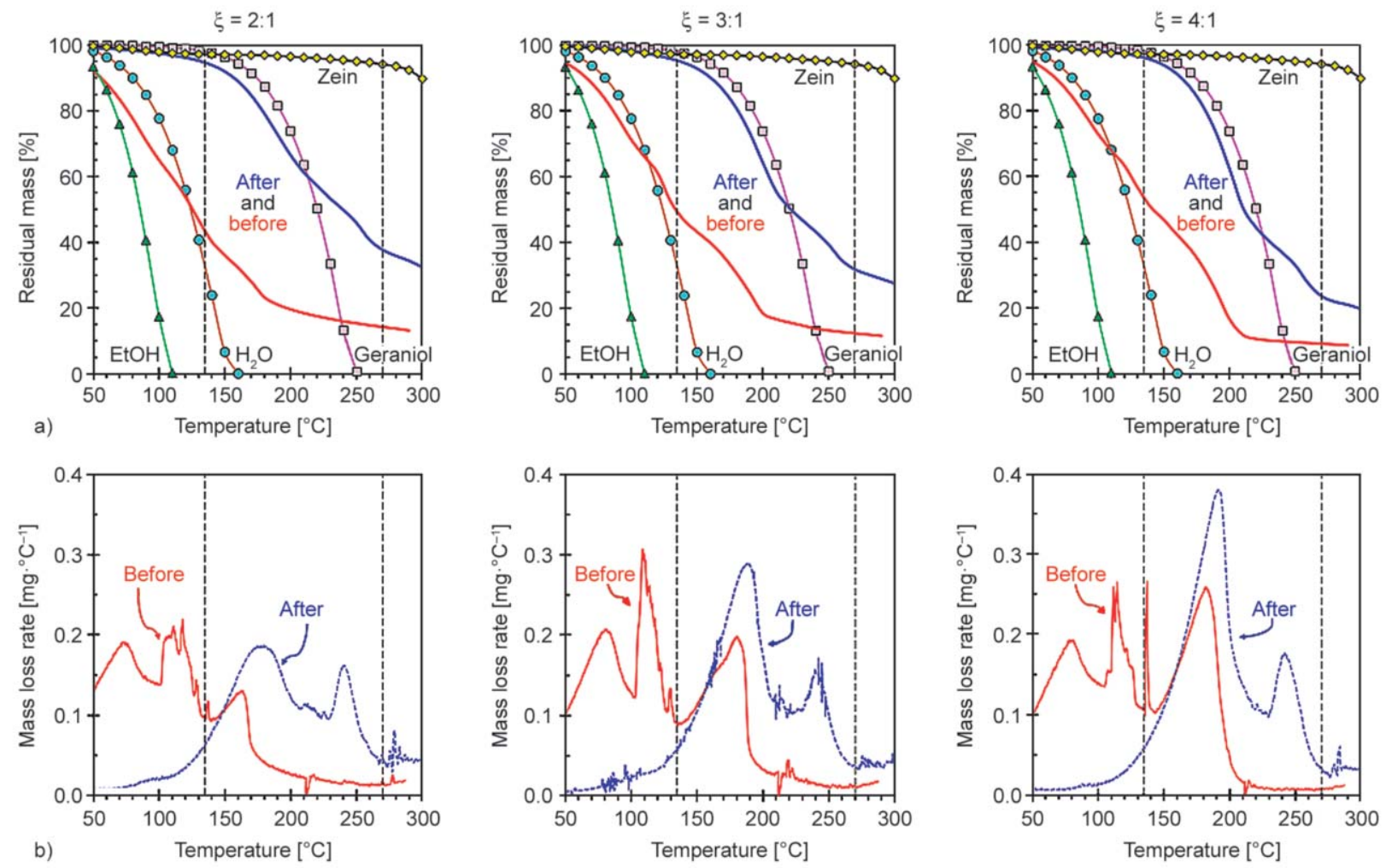

Figure 4. TGA response curves for $\xi$-values where microcapsules were formed. (a) mass loss curves of pure components as well as that of capsules before and after lyophilization, (b) derivative weight curves of capsules before (solid red lines) and after lyophilization (serrated blue lines). Vertical lines indicate the temperature range associated with geraniol mass loss. 
Table 2. Effect of geraniol-to-zein mass ratio on particle yield, estimated encapsulation efficiency and zein participation efficiency.

\begin{tabular}{|l|c|c|c|}
\hline $\begin{array}{c}\text { Geraniol-to-zein mass } \\
\text { ratio, } \boldsymbol{\xi} \\
{[-]}\end{array}$ & $\mathbf{2}$ & $\mathbf{3}$ & $\mathbf{4}$ \\
\hline Microcapsule yield & $79.5 \pm 4.2$ & $85.3 \pm 1.8$ & $80.2 \pm 2.9$ \\
\hline Encapsulation efficiency \\
\hline \begin{tabular}{l|l|l|}
$|l|$ \\
\hline - in wet state
\end{tabular} & $76.7 \pm 4.2$ & $85.3 \pm 2.6$ & $82.3 \pm 3.6$ \\
\hline Zein participation lyophilization & $75.1 \pm 4.3$ & $83.5 \pm 3.3$ & $78.9 \pm 3.4$ \\
\hline - in wet state & $85.1 \pm 5.1$ & $87.5 \pm 5.9$ & $71.6 \pm 3.9$ \\
\hline - after lyophilization & $88.2 \pm 5.9$ & $91.6 \pm 7.2$ & $85.4 \pm 3.9$ \\
\hline
\end{tabular}

amount of ethanol and water. These encapsulated fractions are released at higher temperatures than the range associated with the volatilization of the respective pure components. The absence of these peaks in the derivative weight curves of lyophilized capsules, shown as serrated lines on Figure 4b, indicates that even encapsulated ethanol and water were removed by the lyophilization process. The second peak observed in the responses measured after lyophilization, is in the temperature range where the boiling point of geraniol $\left(230^{\circ} \mathrm{C}\right)$ is reached and an increase in mass loss rate is to be expected.

The encapsulation efficiencies calculated from particle oil fractions measured both before and after lyophilization are summarized in Table 2 . The estimated zein participation efficiencies are also given. The results confirm that the oil was most effectively encapsulated in the $\xi=3$ system.

\subsection{Scanning electron and confocal microscopy}

The image of the microcapsules in Figure 5 shows that they retained their spherical geometry after lyophilization. However, some agglomeration occurred and in some instances connecting filament-like structures formed between individual particles.

Close-up images of microcapsules, including a ruptured or broken microcapsule, are shown in Figure 6. There are clear defects and surface irregularities in the microcapsule structures. Similar surface morphologies were observed by Filippidi et al. [19]. Some were sufficiently severe so as to cause formations of a puncture in the membrane wall, exposing the inner cavity. However, it is not clear what led to the formation of these defects. However, the ruptured microcapsule provides a view of the internal capsule structure. This confirmed the formation of a

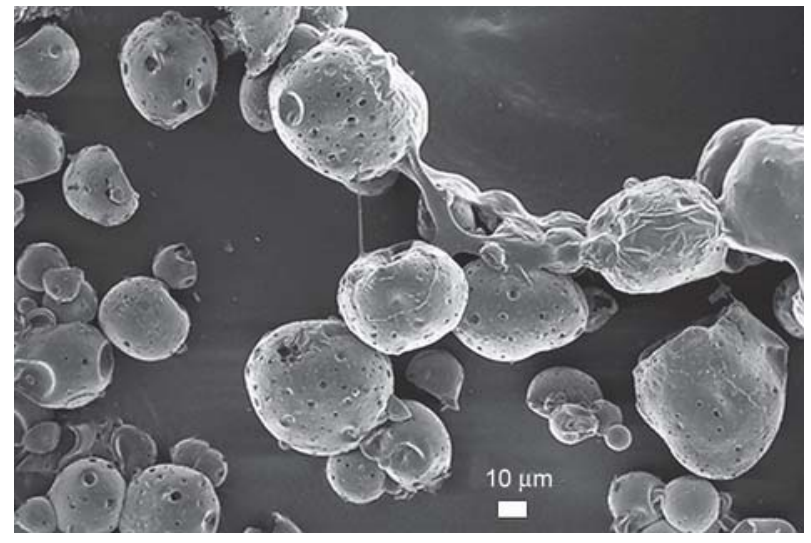

Figure 5. SEM image of multiple microcapsules from the system with a geraniol-to-zein mass ratio of $\xi=3$ after lyophilization.

membrane wall structure enclosing a large hollow internal cavity. Such a structure corresponds to a reservoir system that has the potential to approach zero-order release kinetics if the membrane is in a glassy state.

The microcapsule in Figure $6 \mathrm{~b}$ is approximately the same size as the $d_{50}$ value for this system. According to Table 1, the expected wall thickness is about $1.2 \mu \mathrm{m}$

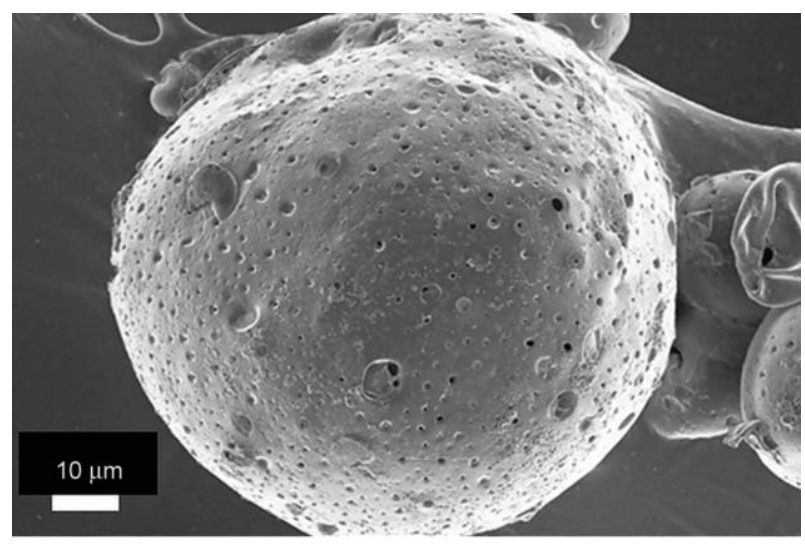

a)

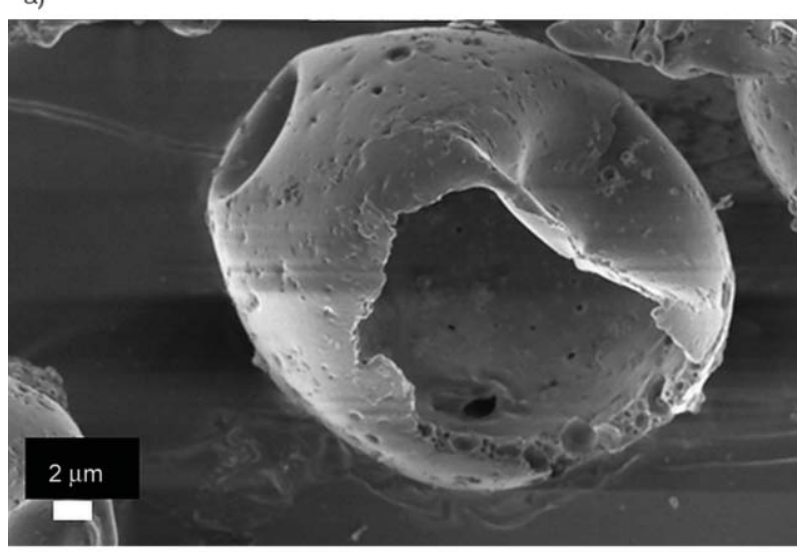

b)

Figure 6. SEM images of capsules from the geraniol-to-zein 3:1 system after lyophilization. (a) Intact and, (b) a ruptured capsule. 
but, in actual fact, the real value is closer to double this value. The discrepancy is attributed to the porous, closed-cell foam-like nature of the microcapsule walls.

Whether this structure is only limited to the ruptured microcapsules due to the possible collapse of the internal structure upon rupturing during lyophilization, or whether it extends to microcapsules prior to lyophilization, was clarified by confocal microscopy. Confocal images of microcapsules $(\xi=3)$ before and after lyophilization are shown in Figure 7. The internal structures observed under confocal microscopy correspond well to those observed under the SEM. There is a well-defined core cavity in all the microcapsules, with surrounding smaller cavities visible inside the fluorescing membrane walls. The membrane

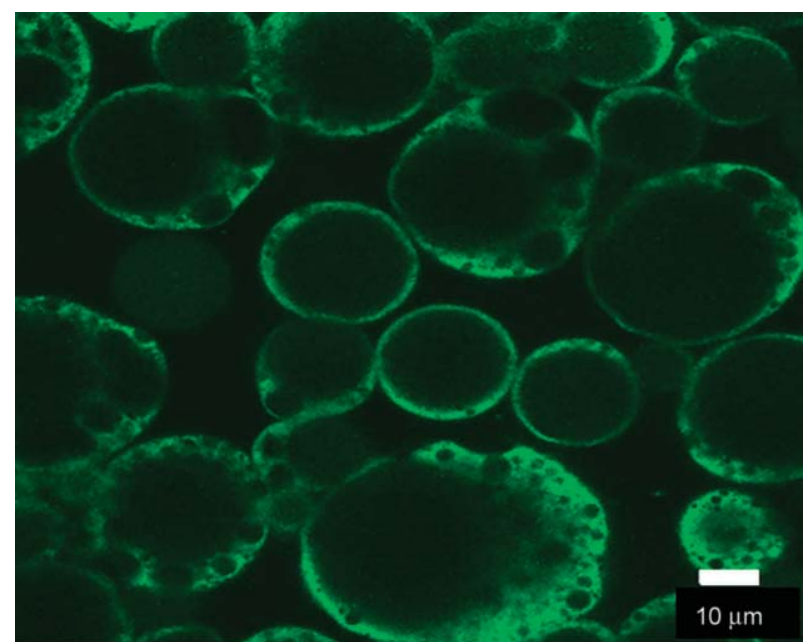

a)

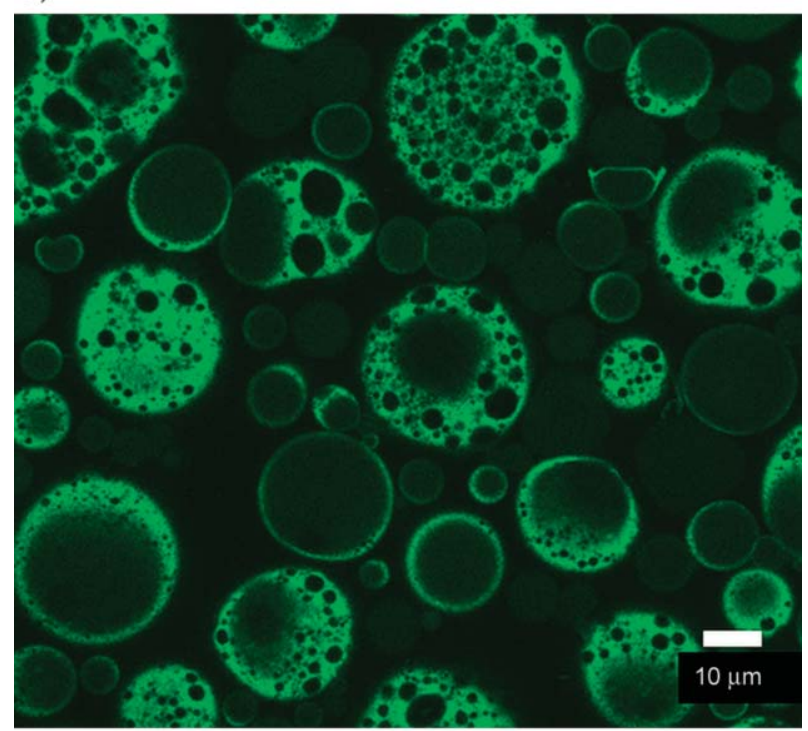

b)

Figure 7. Confocal image of the internal structure of multiple microcapsules with $\xi=3$, (a) before and (b) after lyophilization. cavities, however, can vary significantly in size between microcapsules. Except for some agglomeration observed in the image taken after lyophilization, the particles exhibit the same morphology.

\subsection{Release kinetics}

The release kinetics of the geraniol-to-zein $\xi=3$ system, at different temperatures, is shown in Figure 8 as the fraction geraniol released. These results, in the range where 5 to $95 \mathrm{wt} \%$ of geraniol was vaporized, were fitted with the semi-empirical power law model [22] (Equation (4)):

$\frac{m_{\mathrm{r}}(t)}{m_{\mathrm{o}}}=\left(\frac{t}{\tau}\right)^{\mathrm{n}}$

where $m_{\mathrm{r}}(\mathrm{t})$ is the mass geraniol released at time $t$; $m_{\mathrm{o}}$ the original mass of encapsulated geraniol; $t$ is the time; $\tau$ is a characteristic time constant and $n$ is the release exponent. For a reservoir system with a spherical geometry with Fickian diffusion through the wall rate-limiting, the expected value for $n$ equals 0.85 [22]. However, the best fit value for the release exponent for the present data set was $n=0.80$.

The parameter $\tau$ is related to an effective diffusion coefficient $D_{\mathrm{E}}$ and a characteristic diffusion path length $L$ via the expression $\tau \propto L^{2} / D_{\mathrm{E}}$. This means that it is expected to follow an Arrhenius type temperature dependence:

$\tau=\tau_{0} \mathrm{e}^{\frac{E}{R T}}$

where $\tau_{0}$ is a pre-exponential factor, $E$ is the activation energy, $R$ is the gas constant and $T$ is the

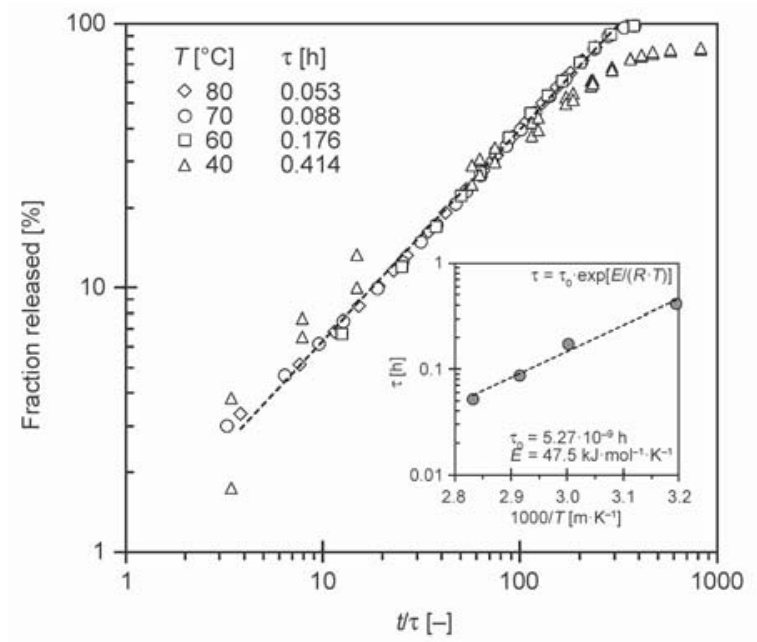

Figure 8. Fraction of encapsulated geraniol released for the $\xi=3$ system at different isothermal temperatures as fitted with Equations (4) and (5). 
absolute temperature. The present data are consistent with an activation energy of $47.5 \mathrm{~kJ} \cdot \mathrm{mol}^{-1} \cdot \mathrm{K}^{-1}$.

\section{Conclusions}

The limited solubility window of zein in waterethanol mixtures was exploited to micro-encapsulate geraniol in a one-step process conducted at ambient conditions. High encapsulation efficiencies and good particle yields were obtained at geraniol to zein mass ratios above unity, but very poor yields were obtained at lower ratios. This was explained in terms of the variation in the relative positions of the phase separation boundaries, for geraniol and zein respectively, as their mass ratio in the starting solution is changed. The highest microcapsule yield and efficiency were obtained at a geraniol-to-zein ratio of 3 . The near spherical microcapsules formed in this system featured a closed-cell foamed membrane wall that encloses a large internal cavity. The particle size distribution was log-normal with $d_{10}, d_{50}$ and $d_{90}$ values of 10,32 and $88 \mu \mathrm{m}$ respectively. The release rate followed power law kinetics with a release exponent of 0.80 .

\section{Acknowledgements}

This work is based on the research supported by the National Research Foundation (NRF) of South Africa (Grant No. 92781). Any opinion, finding and conclusion or recommendation expressed in this material is that of the author(s) and the NRF does not accept any liability in this regard.

\section{References}

[1] Villaverde J. J., Sevilla-Morán B., López-Goti C., Alonso-Prados J. L., Sandín-España P.: Trends in analysis of pesticide residues to fulfil the European Regulation (ec) no. 1107/2009. TrAC Trends in Analytical Chemistry, 80, 568-580 (2016). https://doi.org/10.1016/j.trac.2016.04.017

[2] de Oliveira J. L., Campos E. V. R., Bakshi M., Abhilash P. C., Fraceto L. F.: Application of nanotechnology for the encapsulation of botanical insecticides for sustainable agriculture: Prospects and promises. Biotechnology Advances, 32, 1550-1561 (2014). https://doi.org/10.1016/j.biotechadv.2014.10.010

[3] Koul O., Walia S., Dhaliwal G. S.: Essential oils as green pesticides: Potential and constraints. Biopesticides International, 4, 63-84 (2008).
[4] Prinsloo G., Ninkovic V., van der Linde T., van der Westhuizen A. J., Pettersson J., Glinwood R.: Test of semiochemicals and a resistant wheat variety for russian wheat aphid management in South Africa. Journal of Applied Entomology, 131, 637-644 (2007). https://doi.org/10.1111/j.1439-0418.2007.01213.x

[5] El Asbahani A., Miladi K., Badri W., Sala M., Ait Addi E. H. A., Casabianca H., El Mousadik A., Hartmann D., Jilale A., Renaud F. N. R., Elaissari A.: Essential oils: From extraction to encapsulation. International Journal of Pharmaceutics, 483, 220-243 (2015). https://doi.org/10.1016/j.ijpharm.2014.12.069

[6] Allsopp E., Prinsloo G. J., Smart L. E., Dewhirst S. Y.: Methyl salicylate, thymol and carvacrol as oviposition deterrents for Frankliniella occidentalis (pergande) on plum blossoms. Arthropod-Plant Interactions, 8, 421427 (2014).

https://doi.org/10.1007/s11829-014-9323-2

[7] Kakran M., Antipina M. N.: Emulsion-based techniques for encapsulation in biomedicine, food and personal care. Current Opinion in Pharmacology, 18, 47-55 (2014). https://doi.org/10.1016/j.coph.2014.09.003

[8] Bakry A. M., Abbas S., Ali B., Majeed H., Abouelwafa M. Y., Mousa A., Liang L.: Microencapsulation of oils: A comprehensive review of benefits, techniques, and applications. Comprehensive Reviews in Food Science and Food Safety, 15, 143-182 (2016). https://doi.org/10.1111/1541-4337.12179

[9] Sansukcharearnpon A., Wanichwecharungruang S., Leepipatpaiboon N., Kerdcharoen T., Arayachukeat S.: High loading fragrance encapsulation based on a polymer-blend: Preparation and release behavior. International Journal of Pharmaceutics, 391, 267-273 (2010). https://doi.org/10.1016/j.ijpharm.2010.02.020

[10] Chen W., Viljoen A. M.: Geraniol - A review of a commercially important fragrance material. South African Journal of Botany, 76, 643-651 (2010). https://doi.org/10.1016/j.sajb.2010.05.008

[11] Shukla R., Cheryan M.: Zein: The industrial protein from corn. Industrial Crops and Products, 13, 171-192 (2001). https://doi.org/10.1016/S0926-6690(00)00064-9

[12] Patel A. R., Velikov K. P.: Zein as a source of functional colloidal nano- and microstructures. Current Opinion in Colloid and Interface Science, 19, 450-458 (2014). https://doi.org/10.1016/j.cocis.2014.08.001

[13] Wang L-J., Hu Y-Q., Yin S-W., Yang X-T., Lai F-R., Wang S-Q.: Fabrication and characterization of antioxidant pickering emulsions stabilized by zein/chitosan complex particles (ZCPs). Journal of Agricultural and Food Chemistry, 63, 2514-2524 (2015). https://doi.org/10.1021/jf505227a 
[14] Parris N., Cooke P. H., Hicks K. B.: Encapsulation of essential oils in zein nanospherical particles. Journal of Agricultural and Food Chemistry, 53, 4788-4792 (2005). https://doi.org/10.1021/jf040492p

[15] Wang Y., Su C-P., Schulmerich M., Padua G. W.: Characterization of core-shell structures formed by zein. Food Hydrocolloids, 30, 487-494 (2013). https://doi.org/10.1016/j.foodhyd.2012.07.019

[16] Chen H., Zhong Q.: A novel method of preparing stable zein nanoparticle dispersions for encapsulation of peppermint oil. Food Hydrocolloids, 43, 593-602 (2015). https://doi.org/10.1016/j.foodhyd.2014.07.018

[17] da Rosa C. G., de Oliveira Brisola Maciel M. V., de Carvalho S. M., de Melo A. P. Z., Jummes B., da Silva T., Martelli S. M., Villetti M. A., Bertoldi F. C., Barreto P. L. M.: Characterization and evaluation of physicochemical and antimicrobial properties of zein nanoparticles loaded with phenolics monoterpenes. Colloids and Surfaces A: Physicochemical and Engineering Aspects, 481, 337-344 (2015).

https://doi.org/10.1016/j.colsurfa.2015.05.019
[18] Lee S. J., Rosenberg M.: Preparation and properties of glutaraldehyde cross-linked whey protein-based microcapsules containing theophylline. Journal of Controlled Release, 61, 123-136 (1999).

https://doi.org/10.1016/S0168-3659(99)00108-X

[19] Filippidi E., Patel A. R., Bouwens E. C. M., Voudouris P., Velikov K. P.: Microcapsules: All-natural oil-filled microcapsules from water-insoluble proteins. Advanced Functional Materials, 24, 5962-5968 (2014). https://doi.org/10.1002/adfm.201400359

[20] Li H., Zhang T., Fu W., Tamura K.: Liquid-liquid phase behaviors of geraniol in aqueous alcohol mixtures. Journal of Chemical and Engineering Data, 57, 148154 (2012). https://doi.org/10.1021/je200940a

[21] Ouali L., Léon G., Normand E., Johnsen H., Dyrli A., Schmid R., Benczedi D.: Mechanism of Romascone ${ }^{\circledR}$ release from hydrolyzed vinyl acetate nanoparticles: Thermogravimetric method. Polymers for Advanced Technologies, 17, 45-52 (2006). https://doi.org/10.1002/pat.702

[22] Siepmann J., Peppas N. A.: Modeling of drug release from delivery systems based on hydroxypropyl methylcellulose (HPMC). Advanced Drug Delivery Reviews, 48, 139-157 (2001).

https://doi.org/10.1016/S0169-409X(01)00112-0 